\title{
The Discovery of Two Nearby Carbon Dwarfs
}

\author{
Patrick J. Lowrance ${ }^{1}$, J. Davy Kirkpatrick ${ }^{1}$, I. Neill Reid ${ }^{2}$, Kelle L. Cruz ${ }^{3}$, \& James \\ Liebert $^{4}$
}

\begin{abstract}
The comparison of optical and 2MASS near-infrared photometry for large samples of catalogued proper motion stars has the potential to discover previously unrecognized nearby objects of rare type. In this paper we present the discovery of two new carbon dwarfs, LSR 2105+2514 and LP 758-43, which were drawn from proper motion lists and which lie in a sparsely populated part of optical/near-IR color-color space. Their optical spectra, exhibiting absorptions by $\mathrm{C}_{2}$ and/or CN, are discussed. LSR $2105+2514$ is believed to lie within $200 \mathrm{pc}$ and would have $M_{K_{s}} \geq 6.7$, making it lower in luminosity than any carbon dwarf with a measured trigonometric parallax. LP 758-43, which is not as red but still probably cooler than the best studied carbon dwarfs, is believed to lie within 360 pc. Using our optical/near-infrared selection technique on published lists of proper motion stars, we hope in the near future to expand further the current sample of carbon dwarfs, which numbers only 31 objects at this writing.
\end{abstract}

Subject headings: Stars: atmospheres — stars:carbon — techniques: photometric

\section{Introduction}

The distinctive optical spectra of carbon giants have led to their use as probes of the Galactic halo, especially since their bright magnitudes enable radial velocities to be obtained at very large distances (Aaronson 1983). For the first three quarters of the last century,

\footnotetext{
${ }^{1}$ Infrared Processing and Analysis Center, California Institute of Technology, Pasadena, CA; lowrance,davy@ipac.caltech.edu

${ }^{2}$ Space Telescope Science Institute, Balitmore, MD; inr@stsci.edu

${ }^{3}$ University of Pennsylvania, Philadelphia, PA; kelle@hep.upenn.edu

${ }^{4}$ University of Arizona, Tucson, AZ; liebert@as.arizona.edu
} 
giants were the only luminosity class of carbon stars known. With the discovery of G77-61 (Dahn et al. 1977) at a mere 58 pc and therefore relatively low luminosity, a category of dwarf carbon $(\mathrm{dC})$ stars was finally recognized. This, however, presented a puzzle because a low mass star is incapable of the helium fusion needed to create carbon. This puzzle was resolved by invoking the presence of an evolved, second member of the system - now an undetected white dwarf secondary. It was assumed that the second member, as it passed through its asymptotic giant branch (AGB) phase, transferred mass onto a lower mass main sequence star, enriching it in carbon-bearing material (Dahn et al 1977). The lower mass member was proposed to be a metal-poor subdwarf, and therefore the small amounts of oxygen it does contain in the atmosphere will be overwhelmed by the carbon. A decade after this theory was advanced, its credibility was strengthened by the discovery of radial velocity variations in G77-61 (indicating an unseen companion; Dearborn et al 1986), and by discoveries of two other $\mathrm{dC}$ stars in binaires containing a visual white dwarf secondary (Heber et al 1993; Liebert et al 1994). A review of dwarf carbon star research has been given recently by Green (2000).

The process of making a carbon dwarf may also begin to explain the existence of a rare class of carbon giants known as $\mathrm{CH}$ stars. $\mathrm{CH}$ stars are halo members with peculiar abundances, $\mathrm{C} / \mathrm{O}>1$, and a strong overabundance of s-process elements thought to be produced during an AGB phase. Interestingly, they are all in known binary systems, and those with derived mass ratios are consistent with white dwarf secondaries. As such, these giant stars may represent the dC's at a more advanced evolutionary state (McClure 1984; Wallerstein \& Knapp 1998).

Current understanding of dC's is, however, limited by the small number of such objects now recognized. Only as more dC's are found can we fully test the current hypotheses and understand the role of dC's in the overall picture of stellar evolution. Fortunately, large areal photometric surveys are beginning to uncover these objects in larger numbers. Margon et al (2002) have reported the discovery of 39 faint high-latitude carbon stars from the Sloan Digital Sky Survey (SDSS), of which 17 exhibit proper motions exceeding their three-sigma astrometric uncertainties and are assumed to be dC's. In this paper we report the first two dC's uncovered by the Two Micron All Sky Survey (2MASS). 


\section{The First New Carbon Dwarf}

\subsection{Discovery and Spectroscopic Confirmation}

From an analysis of multi-epoch Digitized Sky Survey (DSS) images lying within 25 degrees of the Galactic plane, Lépine, Shara \& Rich (2002; hereafter LSR02) published a list of objects having proper motions larger than $0.5^{\prime \prime} / \mathrm{yr}$ and magnitudes down to $\mathrm{R}=19.8$ mag. Their list, although a rediscovery of many objects tabulated in the Luyten Half-Second Catalogue (Luyten 1979), also included 141 new proper motion stars not recognized previously. Approximately forty percent of those objects fall in the 2MASS Second Incremental Data Release (Cutri et al 2000), so their characteristics can be studied via diagrams employing the $\mathrm{B}$ and $\mathrm{R}$ data from the DSS as well as the $\mathrm{JHK}_{s}$ data from 2MASS. In looking at the colors of these objects we noticed that one, LSR2105+2514 (21:05:16:58,+25:14:48.1; J2000), has a very red $\mathrm{J}-\mathrm{K}_{s}$ color (1.28) like a late-M or early-L dwarf (Table 1) but an R-J color (1.6) that is far too blue for such a dwarf. A finder chart for this object is shown in LSR02.

We observed LSR 2105+2514 spectroscopically on 07 Aug 2002 UT using the Double Spectrograph (Oke \& Gunn 1982) on the Hale 5-meter telescope at Palomar Observatory. Our instrumental setup used the D68 dichroic to split the light between the two channels at $\sim 6800 \AA$. A 300 lines/mm grating was used in the blue arm and a 316 lines $/ \mathrm{mm}$ grating in the red arm with grating tilts that provided a few hundred Angstroms of duplicate coverage between the two arms. This provided continuous wavelength coverage from 3800 to $9100 \AA$. Use of a 2 ". 0 slit resulted in a resolution of $8 \AA$. Observations were reduced using standard techniques following Kirkpatrick, Henry, \& McCarthy (1991) and flux calibrated using the standard star Feige 110 (Hamuy et al 1994).

The resulting spectrum is shown in Figure 1. The strong bands of $\mathrm{C}_{2}$ at 4382,4737 , 5165, 5636, and $6191 \AA$ are easily recognizable, indicating that this is a carbon star. The high proper motion measured by LSR02 of $0.563^{\prime \prime} / \mathrm{yr}$ along with its magnitudes of $\mathrm{B}=18.7 \pm 0.5$ and $\mathrm{R}=16.1 \pm 0.5$ indicate that LSR $2105+2514$ is a dwarf star and not a background giant.

\subsection{Known Carbon Dwarfs and a Comparison to LSR 2105+2514}

In a given volume of space, carbon dwarfs are believed to greatly outnumber carbon giants and as such should be the dominant type of carbon star in the Galaxy (Green 2000). Nonetheless, few dC's are catalogued. Before the $17 \mathrm{dC}$ 's recently announced by Margon et 
al (2002), previous discoveries only dribbled in since the 1970's. Table 1 gives a list of all known dwarf carbon stars known as of this writing.

Only three of the carbon dwarfs in Table 1 have known distances, and the faint magnitudes and presumably larger distances of many of the others may preclude a robust trigonmetric parallax determination. LSR $2105+2514$ is bright enough that it has already been added to the USNO parallax program. Even without a $\pi_{\text {Trig }}$, however, we can place a crude upper limit to its distance based on its measured proper motion of 0 ' $563 / \mathrm{yr}$ and $\theta=150.2 \mathrm{deg}$ (LSR02). Assuming that LSR $2105+2514$ is gravitationally bound in the Galaxy, it cannot exceed an escape velocity of $\sim 500 \mathrm{~km} / \mathrm{s}$ (Carney, Latham, \& Laird 1988). We then calculate expected $(\mathrm{U}, \mathrm{V}, \mathrm{W})$ motions, stepping out in distance assuming $\mathrm{V}_{\text {rad }}=0$. We have transformed those velocities to galactic motions using a solar motion of $(9,11,6)$ and assuming a rotational velocity of $220 \mathrm{~km} / \mathrm{s}$ for the Local Standard of Rest. LSR $2105+2514$ has a total velocity exceeding $500 \mathrm{~km} / \mathrm{s}$ if the distance exceeds 200 parsecs. That suggests that LSR2105+2514 has $\mathrm{M}_{K s} \geq 6.7$ mag. This would indicate a lower luminosity carbon dwarf than any of the ones with a measured $\pi_{\text {Trig }}$ (see Table 1). This suggestion is seemingly supported by its red $\mathrm{J}-\mathrm{K}_{s}$ color of 1.28 , which indicates it is also one of the coolest carbon dwarfs.

\section{Finding Other Carbon Dwarfs}

Currently, the only way to distinguish between a carbon dwarf and a carbon giant is through its luminosity, and hence one needs a measure of the parallax or an indirect distance indicator such as proper motion. To date, discriminators based on spectroscopy or photometry have been proposed, but none has met with $100 \%$ success.

To define a list of carbon dwarf candidates, we have chosen the list of 36,085 NLTT objects (Luyten 1980) that Gould \& Salim (2002) have cross-referenced against the 2MASS 2nd Incremental Data Release. If we plot known carbon dwarfs on an $\mathrm{R}-\mathrm{J}$ vs. J-K diagram along with objects from the NLTT, we find that carbon dwarfs generally have much redder $\mathrm{J}-\mathrm{K}_{s}$ colors than the cloud of main sequence stars at similar $\mathrm{R}-\mathrm{J}$ colors (Figure 2). Examining the infrared spectra of carbon dwarfs in Joyce (1998), we find shallow CN bands at $\mathrm{R}$ and $\mathrm{K}$ along with the $\mathrm{K}$-band $\mathrm{CO}$ absorptions that are normally in low-mass stars, but a defining characteristic of these carbon dwarfs are large CN absorption bands at $\mathrm{J}$. This results in bluer $\mathrm{R}-\mathrm{J}$ and redder $\mathrm{J}-\mathrm{K}_{s}$ colors than main-sequence objects of similar temperature. Exploring objects from the NLTT in this part of color space should increase the sample size of known carbon dwarfs. 
Objects with infrared colors are often placed on $(\mathrm{J}-\mathrm{H}) /\left(\mathrm{H}-\mathrm{K}_{s}\right)$ to decipher between the giant and dwarf populations. Unfortunately, as Margon et al 2002 comments and Figure 3 demonstrates, some of the carbon dwarfs have $\mathrm{J}-\mathrm{H}$ colors like giants, and therefore, cannnot be distinguished using this diagram alone. Likewise, there are a few unusual giants that fall in the lower half of the diagram along with the bulk of the dwarfs. It is therefore important to search for spectroscopic indicators that might help us understand if these differences are due to temperature or abundance effects and possibly differentiate carbon dwarfs from carbon giants.

\section{A Second New Carbon Dwarf}

Using the R-J vs. $\mathrm{J}-\mathrm{K}_{s}$ selection criteria outlined above and in the caption to Figure 2, we have identified and observed several NLTT carbon dwarf candidates. LP 758-43, with $\mathrm{R}-\mathrm{J}=2.0$ and $\mathrm{J}-\mathrm{K}_{s}=1.10$, was observed on 2002 Sep $26 \mathrm{UT}$ with the Multi-Aperture Red Spectrograph (MARS) on the KPNO Mayall 4m telescope. We used the VB8050-450 grating and $2.0^{\prime \prime}$-wide long slit, covering the wavelength range 5600 to $10000 \AA$ at a resolution of $\sim 9 \AA$. The data were reduced and wavelength calibrated using standard techniques in IRAF, and flux calibrated through observations of the spectrophotometric standard Feige 110. The spectrum of LP 758-43 is plotted in Figure 1 and compared with other known carbon dwarfs. The CN bands appear very similar in depth to the previously known carbon dwarfs. A finding chart for LP 758-43 is presented in Figure 4. Other candidates which turned out to be close doubles, mismatches with reddened stars, a white dwarf/red dwarf binary and M dwarfs will be summarized in the complete survey (Lowrance et al 2003, in prep).

With $\mathrm{K}_{s}=11.90$, LP 758-43 is one of the brighter examples of a $\mathrm{dC}$ (see Table 1), enabling future trigonometric parallax measurements. Without a $\pi_{\text {Trig }}$, we can attempt to place a crude upper limit to the distance using the arguments used for LSR $2105+2514$. For its measured proper motion of $0.255^{\prime \prime} / \mathrm{yr}$ and $\theta=90.4$ not to exceed the escape velocity of $\sim 500 \mathrm{~km} / \mathrm{s}$, its distance would be no greater than $\sim 360 \mathrm{pc}$ with a $\mathrm{M}_{K_{s}}>4.1 \mathrm{mag}$. The $\mathrm{J}_{-} \mathrm{K}_{s}$ color of LP $758-43\left(\mathrm{~J}-\mathrm{K}_{s}=1.1\right)$ is redder than the average $\mathrm{J}-\mathrm{K}_{s}$ color $\left(\mathrm{J}-\mathrm{K}_{s}=0.95\right)$ of the three carbon dwarfs with known distances. Those three have an average $\mathrm{M}_{K_{s}}=6.5$

mag, so if we assume LP 758-43 has a similar or fainter absolute magnitude at $\mathrm{K}_{s}$, it is more likely located at less than 120 pc. 


\section{Conclusions}

We present the optical spectra of two newly discovered carbon dwarfs, LSR $2105+2514$ and LP 758-43, with proper motions of $0.563^{\prime \prime} / \mathrm{yr}$ and $0.255^{\prime \prime} / \mathrm{yr}$, respectively. Both objects were selected based on their unusual optical to infrared colors and lie in a locus on the optical/ near-infrared color-color diagram populated by other known carbon dwarfs. Looking through proper motion catalogs - where it is assured that faint objects are nearby dwarfs and not background giants - for objects of similar color will allow us to produce lists of carbon dwarf candidates for spectroscopic follow-up. Finding more carbon dwarfs, of which only 31 are currently known, can help us understand the role of dC's in stellar evolution. Finally, broadening the sample will allow a search for spectroscopic discriminants that could help distinguish between carbon dwarfs and carbon giants.

P.J.L. acknowledges support from a National Research Council Fellowship. P.J.L. and J. D. K. acknowledge the support of the Jet Propulsion Laboratory, California Institute of Technology, which is operated under contract with the National Aeronautics and Space Administration. KLC acknowledges support from a NSF Graudate Student Fellowship. We are grateful to the referee H. Harris for a careful review of the manuscript and suggested improvements. P.J.L. acknowledges great support from the Palomar Observatory staff. This publication makes use of data from the Two Micron All-Sky Survey, which is a joint

project of the University of Massachusetts and the Infrared Processing and Analysis Center, funded by the National Aeronautics and Space Administration and the National Science Foundation.

\section{REFERENCES}

Aaronson, M. 1983 ApJ 266 L11

Bessell. M. \& Brett, J.M. 1988 PASP 1001134

Carney, B. Latham, D., \& Laird, J.B. 1988 AJ 96560

Cutri, R., et al 2000 http://www.ipac.caltech.edu/2mass/releases/second/doc/explsup.html Dahn, C.C., Liebert,J., Kron, R.G, Spinrad,H., \& Hintzen,P.M. 1977 ApJ, 216757

Dearborn,D.S., Liebert,J., Aaronson,M., Dahn, C.C., Harrington, R.S., Mould, J., \& Greenstein, J.L., 1986 ApJ 300314

Green, P. 2000, The Carbon Star Phenomenon, Proceedings of the 177th Symposium of the International Astronomical Union, held in Antalya, Turkey, May 27-31, 1996. Ed. Robert F. Wing, 2000., p.27 
Green, P. \& Margon, B. 1994 ApJ 423723

Green, P., Margon, B., Anderson, S.F., MacConnell, D. J., 1992 ApJ 400659

Gould, A. \& Salim, S. 2002 ApJ in press

Harris, H.C., Dahn, C.C., Walker R.L., Luginbuhl C.B., Monet A.K.B., Guetter H.H., Stone R.C., Vrba F.J., Monet D.G., Pier J.R. 1998 ApJ 502437

Hamuy, M., Suntzeff, N. B., Heathcote, S. R., Walker, A. R., Gigoux, P., \& Phillips, M. M. 1994 PASP 106566

Heber, U., Bade, N., Jordan, S., \& Voges, W. 1993 A\&A 267 L31

Kirkpatrick, J.D., Henry, T., \& McCarthy, D. 1991 ApJS 77417

Kirkpatrick, J.D. 1992 PhD thesis, University of Arizona

Lepine, S., Shara, M.S., \& Rich, R.M. ApJ 20021241190

Liebert,J., Schmidt,G., Lesser,M., Stepanian,J.A., Lipovetsky,V.A., Chaffee,F., Foltz,C., \& Bergeron, P. 1994 ApJ 421733

Luyten, W.J. 1979 LHS Catalogue 2nd Ed; University of Minnesota

Luyten, W.J. 1980 NLTT Catalogue Vol3; University of Minnesota

Margon, B., et al 2002 Astronomical Journal, 124, 1651

Mcclure R.D. 1984 ApJ 280 L31

Oke, J.B. and Gunn, J.E. 1982, PASP, 94586

Ratnatunga, K.U. 1983, PhD thesis, Australian National Observatory

Totten, E.J., Irwin, M.J., \& Whitelock, P.A. 2000 MNRAS 314630

Wallerstein, G. \& Knapp, G. ARA\& A 1998 Vol 36369

Warren,S.J., Irwin,M.J., Evans,D.W., Liebert,J., Osmer, P.S., \& Hewett,P 1993 MNRAS 261185 
Table 1. List of All Known Carbon Dwarfs

\begin{tabular}{|c|c|c|c|c|c|c|c|}
\hline Name & RA (J2000) & Dec $(J 2000)$ & $\mathrm{J}$ & $\mathrm{H}$ & $\mathrm{K}_{s}$ & $\mathrm{~J}-\mathrm{K}_{s}$ & $\operatorname{Ref}^{a}$ \\
\hline LHS 1075 & 002600.2 & -191852 & $12.54 \pm 0.02$ & $11.92 \pm 0.03$ & $11.59 \pm 0.03$ & 0.95 & 2 \\
\hline SDSS J0039373+152911 & 003937.3 & +152911 & $\ldots$ & $\ldots$ & $\cdots$ & & 7 \\
\hline WIE93 0041-295 & 004334.9 & -291808 & $\ldots$ & $\ldots$ & $\ldots$ & & 10 \\
\hline WIE93 0045-259 & 004817.6 & -253838 & $\ldots$ & $\ldots$ & $\ldots$ & & 10 \\
\hline SDSS J0121503+011303 & 012150.3 & +011303 & $15.11 \pm 0.05$ & $14.25 \pm 0.05$ & $13.81 \pm 0.05$ & 1.30 & 7 \\
\hline SDSS J0125267+000449 & 012526.7 & +000449 & $\ldots$ & $\ldots$ & $\ldots$ & & 7 \\
\hline SDSS J0256346-084854 & 025634.6 & -084854 & $\ldots$ & $\ldots$ & $\ldots$ & & 7 \\
\hline G77-61, LHS 1555 & 033238.0 & +015800 & $11.47 \pm 0.02$ & $10.84 \pm 0.02$ & $10.48 \pm 0.02$ & 0.99 & 1 \\
\hline SDSS J0736213+390725 & 073621.3 & +390725 & $16.76 \pm 0.14$ & $16.09 \pm 0.21$ & $15.44 \pm 0.15$ & 1.33 & 7 \\
\hline SDSS J0822514+461232 & 082251.4 & +461232 & $15.50 \pm 0.06$ & $14.68 \pm 0.07$ & $14.48 \pm 0.09$ & 1.02 & 7 \\
\hline SDSS J0826268+470912 & 082626.8 & +470912 & $15.78 \pm 0.07$ & $15.19 \pm 0.08$ & $15.02 \pm 0.11$ & 0.76 & 7 \\
\hline $\mathrm{PG} 0824+289 \mathrm{~B}^{\mathrm{b}}$ & 082705.1 & +284402 & $\ldots$ & $\ldots$ & $\ldots$ & & 4 \\
\hline SDSS J0858533+012243 & 085853.3 & +012243 & $15.82 \pm 0.10$ & $15.11 \pm 0.09$ & $14.39 \pm 0.08$ & 1.43 & 7 \\
\hline SDSS J0900114-003606 & 090011.4 & -003606 & $\ldots$ & $\ldots$ & $\ldots$ & & 7 \\
\hline SDSS J0948587+583020 & 094858.7 & +583020 & $\ldots$ & $\ldots$ & $\ldots$ & & 7 \\
\hline SDSS J1004325+004338 & 100432.5 & +004338 & $\ldots$ & $\ldots$ & $\ldots$ & & 7 \\
\hline CLS 29 & 104006.4 & +354802 & $12.98 \pm 0.03$ & $12.29 \pm 0.03$ & $12.00 \pm 0.02$ & 0.98 & 9 \\
\hline CLS 31 & 105429.6 & +340230 & $15.50 \pm 0.05$ & $15.02 \pm 0.07$ & $14.66 \pm 0.08$ & 0.84 & 2 \\
\hline $\mathrm{KA}-2$ & 111903.9 & -164450 & $13.24 \pm 0.02$ & $12.61 \pm 0.03$ & $12.48 \pm 0.03$ & 0.76 & 8 \\
\hline SDSS J1129504+003345 & 112950.4 & +003345 & $16.47 \pm 0.16$ & $15.69 \pm 0.14$ & $15.52 \pm 0.24$ & 0.95 & 7 \\
\hline SDSS J1147317+003724 & 114731.7 & +003724 & $\ldots$ & $\ldots$ & & & 7 \\
\hline CLS 50 & 122000.8 & +364803 & $14.41 \pm 0.03$ & $13.93 \pm 0.03$ & $13.79 \pm 0.04$ & 0.62 & 3 \\
\hline SDSS J1353330-004039 & 135333.0 & -004039 & $14.61 \pm 0.04$ & $13.80 \pm 0.04$ & $13.61 \pm 0.05$ & 1.00 & 7 \\
\hline SDSS J1421124-004823 & 142112.4 & -004823 & $\ldots$ & $\ldots$ & $\ldots$ & & 7 \\
\hline CBS 311 & 151905.9 & +500703 & $15.56 \pm 0.06$ & $14.75 \pm 0.07$ & $14.16 \pm 0.07$ & 1.40 & 6 \\
\hline SDSS J1537322+004343 & 153732.2 & +004343 & $15.20 \pm 0.05$ & $\geq 14.36$ & $\geq 14.08$ & $\geq 1.12$ & 7 \\
\hline CLS 96, LP 328-57 & 155237.5 & +292802 & $13.80 \pm 0.03$ & $13.20 \pm 0.04$ & $12.88 \pm 0.03$ & 0.92 & 2 \\
\hline WIE93 2048-348 & 205202.6 & -343732 & $16.47 \pm 0.14$ & $15.87 \pm 0.19$ & $15.29 \pm 0.16$ & 1.18 & 10 \\
\hline LSR2105+2514 & 210516.6 & +251448 & $14.48 \pm 0.03$ & $13.77 \pm 0.03$ & $13.20 \pm 0.04$ & 1.28 & 5 \\
\hline LP $758-43$ & 214937.8 & -113828 & $13.00 \pm 0.02$ & $12.26 \pm 0.02$ & $11.90 \pm 0.03$ & 1.10 & 5 \\
\hline SDSS J2302550+005904 & 230255.0 & +005904 & $15.61 \pm 0.06$ & $14.80 \pm 0.06$ & $14.60 \pm 0.08$ & 1.01 & 7 \\
\hline
\end{tabular}

aDiscovery reference:(1)Dahn et al 1977; (2)Green et al 1994; (3) Green et al 1992; (4) Heber et al 1993; (5) this paper; (6) Liebert et al. 1994; (7) Margon et al 2002; (8) Ratnatunga 1983; (9) Totten, Irwin, \& Whitelock 2000; (10) Warren et al. 1992;

${ }^{\mathrm{b}}$ Photometry does not split into two sources in the 2MASS All-Sky Release. Combined photometry is J=12.423 \pm 0.032 ; $\mathrm{H}=11.802 \pm 0.035 ; \mathrm{K}_{s}=11.650 \pm 0.03$

${ }^{\mathrm{c}}$ Photometry is from the 2MASS All-Sky Release. Those without photometry were too faint for 2MASS.

d The carbon dwarfs with determined parallaxes (Harris et al 1998) are G77-61 $\pi_{\text {Trig }}=16.9 \pm 2.2$ mas $\left(\mathrm{M}_{K_{s}}=6.62\right)$, LHS1075 $\pi_{\text {Trig }}=7.96 \pm 0.84$ mas $\left(\mathrm{M}_{K_{s}}=6.09\right)$, and CLS $96 \pi_{\text {Trig }}=4.54 \pm 0.66$ mas $\left(\mathrm{M}_{K_{s}}=6.17\right)$ 


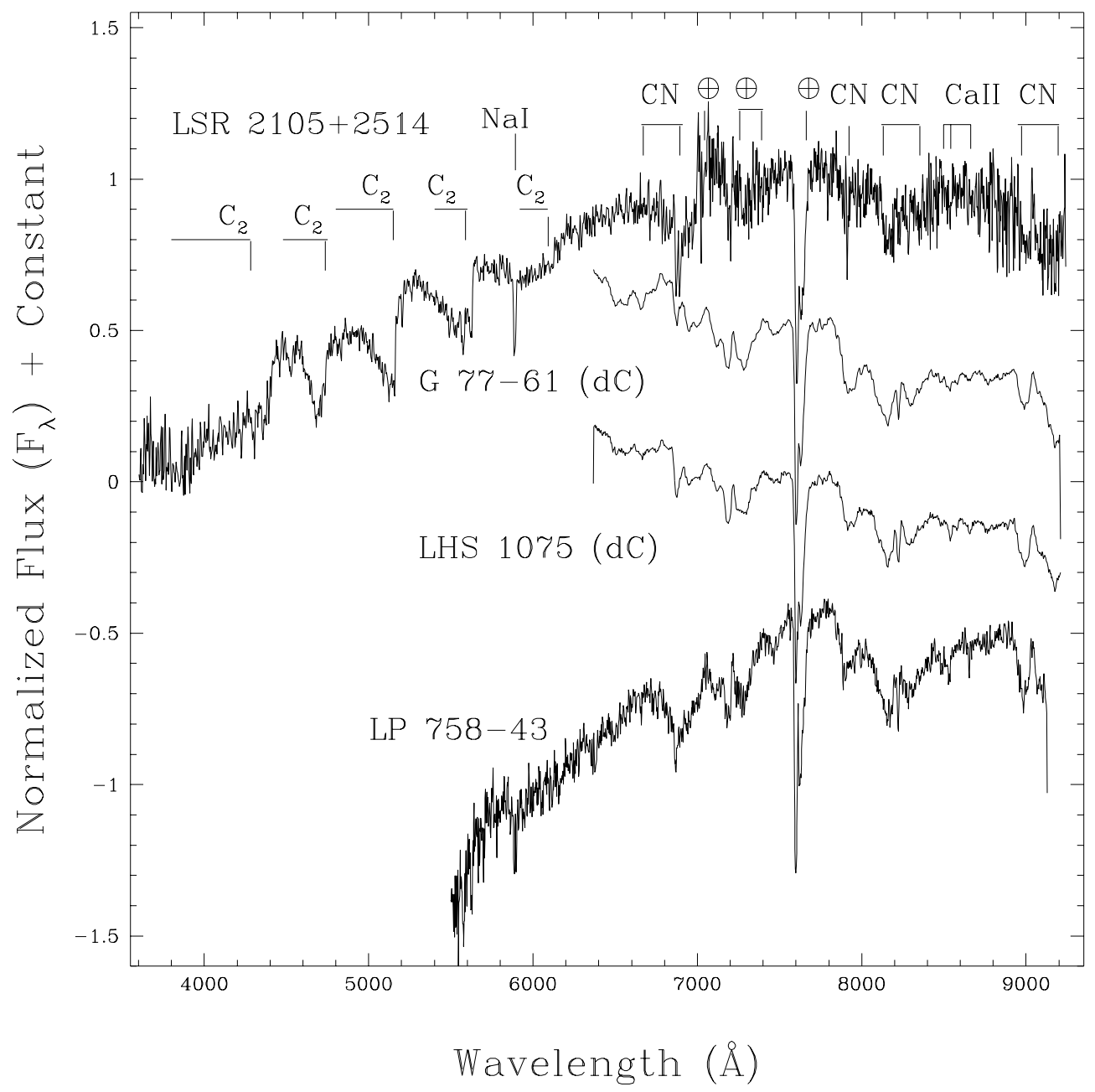

Fig. 1.- Optical spectra of LSR2105+2514 (top) and LP 758-43 (bottom) compared with known carbon dwarfs G77-61 and LHS 1075 from Kirkpatrick (1992). The Swan bands of $\mathrm{C}_{2}$ at $4382,4737,5165$, and $5636 \AA$ are labelled as well as the sharp bandhead of $\mathrm{C}_{2}$ at $6191 \AA$ not often seen in carbon giants. The Na I "D" doublet, Ca II triplet, and the CN bands are also labelled. In the LSR2105+2514 spectrum, there was a small calibration problem in the blueward side of the red channel $(\sim 6800-7200 \AA)$ that caused the flux in that region to be incorrectly elevated. This small portion is suspect, but the remainder of the spectrum should not be affected. The spectra are all normalized at $7500 \AA$, and offsets of $-0.5,-1.0$, and -1.5 have been applied to the normalized flux levels of G77-61, LHS 1075, and LP 758-43, respectively, to separate them from each other in the figure. 


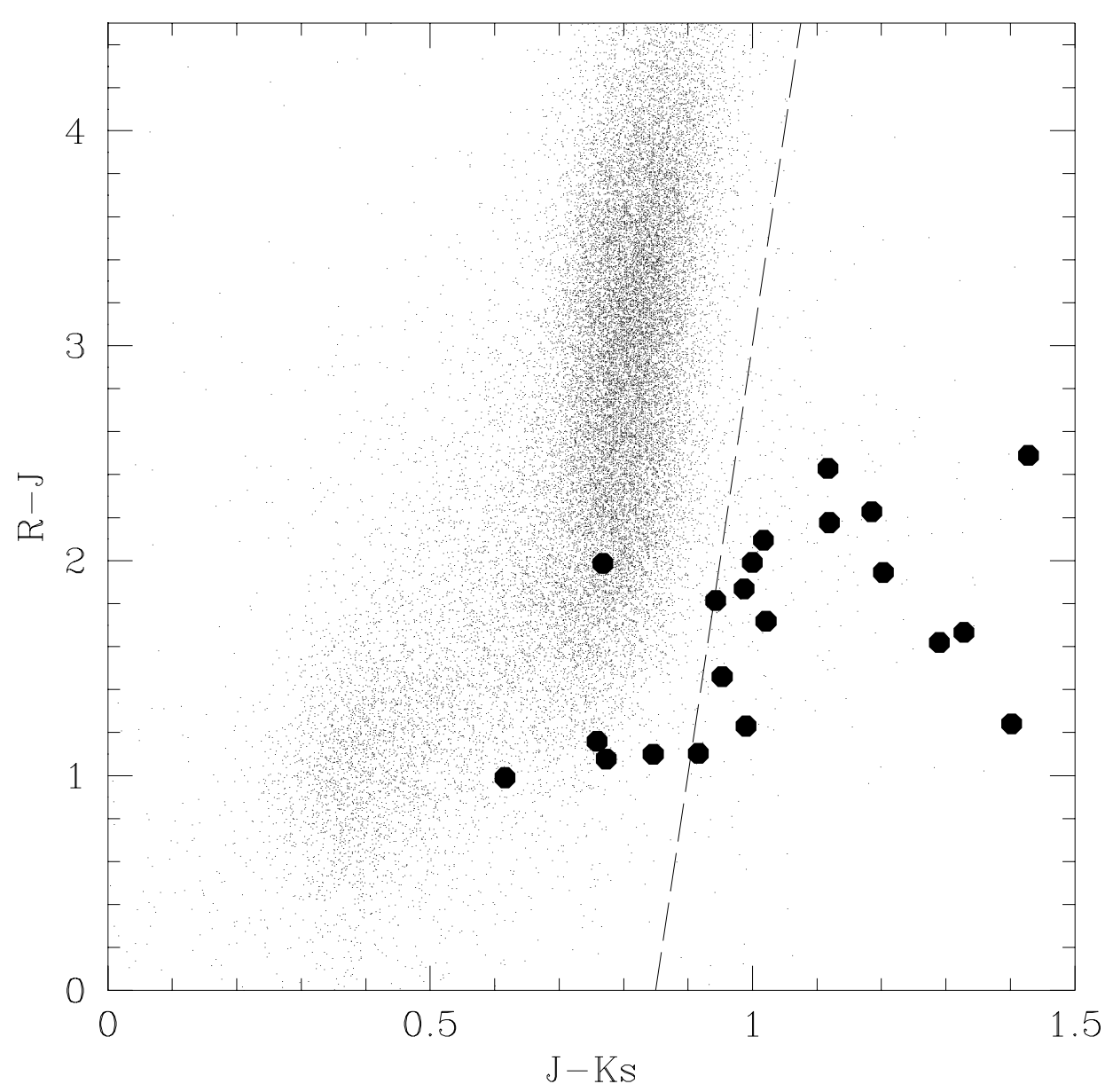

Fig. 2.- R-J vs J-K $\mathrm{K}_{s}$ for stars from Gould \& Salim (2002) with the known carbon dwarfs (filled circles). The dotted line represents a boundary of $(\mathrm{J}-\mathrm{K})=20(\mathrm{R}-\mathrm{J})-17$, used to define a cutoff for the observation selection of possible carbon dwarfs. 


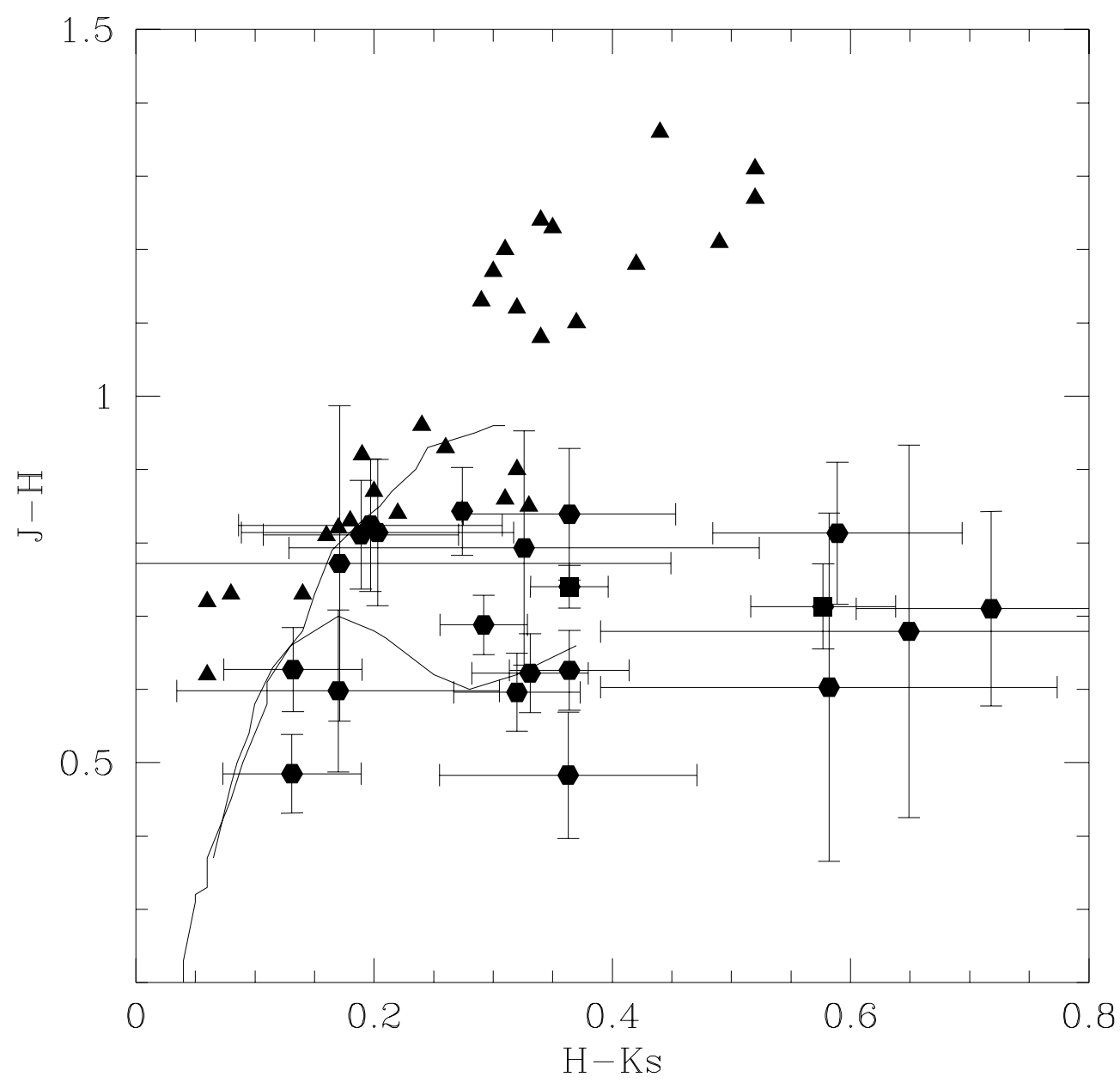

Fig. 3.- $\mathrm{J}-\mathrm{H}$ vs $\mathrm{H}-\mathrm{K}_{s}$ for the two discoveries in this paper (filled squares) and all 2MASS photometered carbon dwarfs (filled hexagons; the two with error bars greater than 0.2 have been omitted here) listed in Table 1 compared with carbon giants (filled triangles). Overplotted are the Bessell \& Brett (1988) tracks for giants (top) and dwarfs (bottom). Notice some of the carbon dwarfs fall within the giant branch of these Bessell-Brett tracks, leading to confusion for objects with small or no proper motion. 


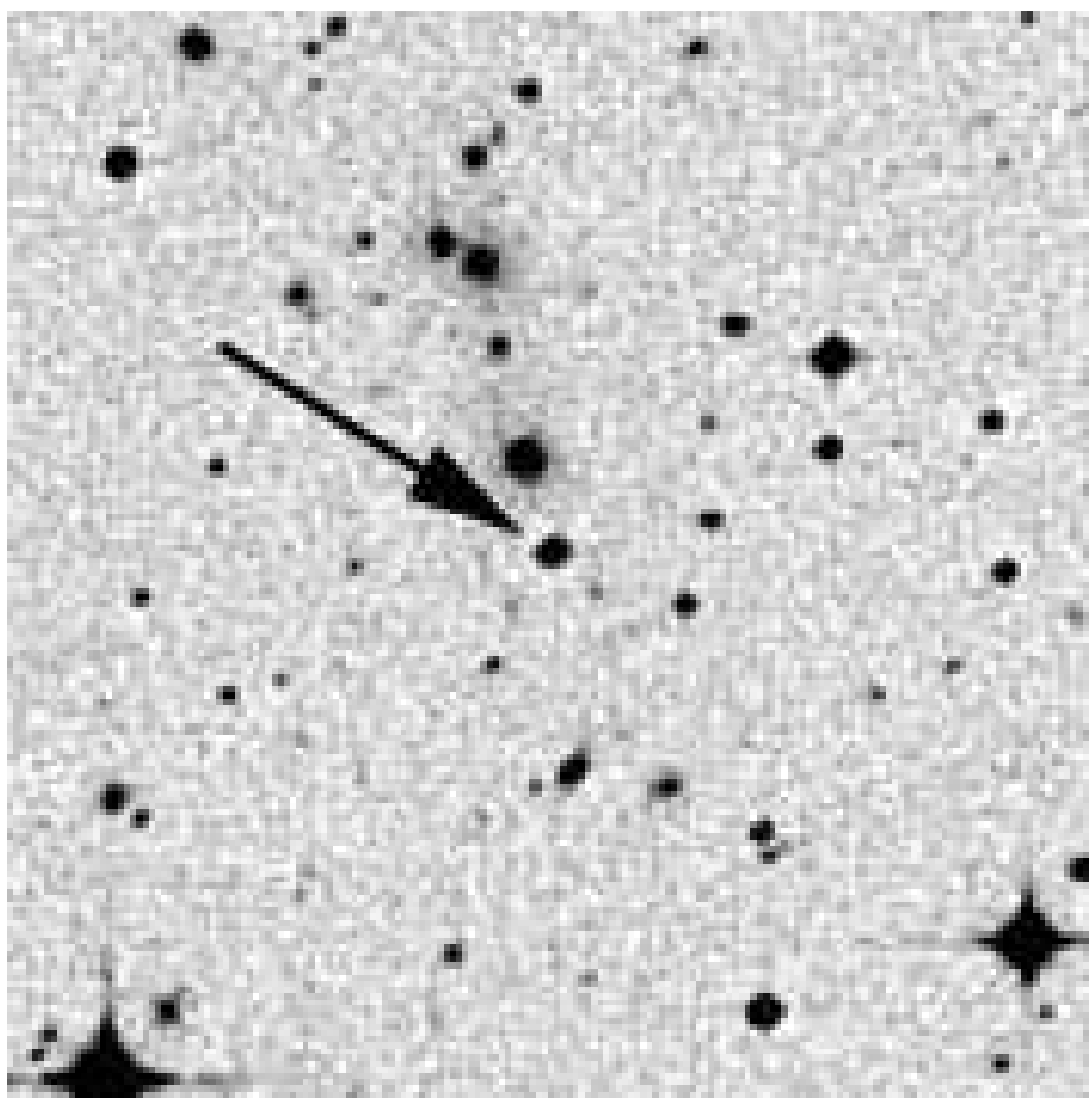

Fig. 4.- Finder chart for LP 758-43. The 5x5 arcminute R-band image from the XDSS (epoch 09 Oct 1988) where an arrow distinguishes the carbon dwarf. 